Martín-Pastor, Andrés, González-Quintial, Francisco. (2021). Surface discretisation with rectifying strips on geodesics. Nexus Network Journal, Vol. 23, no.3 (2021). pp. 565-582 https://doi.org/10.1007/s00004-020-00540-x

\title{
Surface Discretisation with Rectifying Strips on Geodesics
}

\author{
Andrés Martín-Pastor (ETSIE, Dpto. Ingeniería Gráfica, Universidad de Sevilla. \\ Email: archiam@us.es) \\ Francisco González-Quintial, (ETSA, Dpto. Arquitectura, Univ. del País Vasco)
}

\begin{abstract}
The use of geodesic curves of surfaces has enormous potential in architecture due to their multiple properties and easy geometric control using digital graphic tools. Among their numerous properties, the geodesic curves of a surface are the paths along which straight strips can be placed tangentially to the surface. On this basis, a graphical method is proposed to discretize surfaces using straight strips, which optimizes material consumption since rectangular straight strips take advantage of $100 \%$ of the material in the cutting process. The contribution of the article consists of presenting the geometric constraints that characterize this type of panelling from the idea of "rectifying surface", considering the material inextensible. Experimental prototypes that have been part of the research are also described and the final theoretical results are presented.
\end{abstract}

Keywords: Developable surfaces, Rectifying, Geodesic, Discretization, Panelling, Straight Strips

\section{Introduction: On the Discretization of Surfaces by Geodesics}

Geodesic curves have been applied in the design of lightweight structures and gridshells through numerous studies and abundant theorizing (Pirazzi and Weinand 2006; Deregibus and Sassone 2008; Harding et al. 2014; Soriano 2017). This has been made possible thanks to advances in the calculation of geodesics on surfaces using computational environments (Ravi Kumar et al. 2003; Surazhsky et al. 2005; Chen 2010; Lai and Cheng 2011). Apart from its structural use in engineering, the application of these singular curves as architectural envelopes is now being studied. Existing studies on the subject have been carried out using various mathematical tools, such as the notion of conjugate tangents. However, its study can also be approached as a classic problem of descriptive geometry through the concept of a "rectifying surface", a special developable surface that rests on any curve. 
The concept of a rectifying surface, recalled by Snežana Lawrence in "Developable Surfaces: Their History and Application", is due to Gaspard Monge:

His paper, Mémoire sur les développées, les rayons de courbure et les différents genres d'inflexions des courbes à double courbure [Monge 1785; written in 1771, but published in 1785], gave his theory of developable surfaces in an abstract and purely mathematical manner... introducing the rectifying developable, and described such crucial terms in the study of developable surfaces as normal plane, radius of first curvature, and the osculating sphere (Lawrence 2010: 706).

Although rectifying surfaces are a classic concept, highly developed in differential geometry texts (Do Carmo 1976) and collected in treatises of descriptive geometry (Taibo-Fernández 1983; Izquierdo-Asensi 1985), there are very few references in contemporary texts of architectural geometry (Glaeser and Gruber 2007; Pottmann et al. 2007). According to Bo and Wang (2007: 365): "Although the rectifying developable is well known in differential geometry, to the best of our knowledge, it has not been used in practical shape modeling applications". We focus on the precise concept of a rectifying surface to circumscribe a freeform surface by means of strips. The method involves placing rectifying surface strips supported on geodesic curves.

The possibilities offered by strips as developable surfaces have been explored by other authors. A clear explanation of the isometry between the spatial shape and the flat development that occurs in any developable strip has been collected in texts related to curved folding (Kilian et al. 2008; Kilian 2017). The concept of rectifying surface, in turn, is closely related to the pseudo-geodesic as demonstrated in Jiang et al. (2019). However, to the best of our knowledge, no allusion to rectifying surfaces can be found in specific texts that study the problem of panelling a freeform surface using straight strips (Wallner et al. 2010; Kahlert et al. 2011). Neither do rectifying surfaces appear in developable generation models based on developable strips (Liu et al. 2006; Pottmann et al. 2008; Tang et al. 2016), or in contour-based models (Rose et al. 2007; Jung et al. 2015; González-Quintial 2015).

Other work presented by Pottmann et al. (2010) and Deng et al. (2011) addresses how to establish different patterns of these geodetic curves on a freeform surface and describes their possible architectural applications. This is directly related to the aforementioned system of gridshells, which is based on the placement of straight strips on these families of curves.

All these models - and especially the model used in gridshells - start from the premise that a strip can always be placed on the geodesic of a surface, which is true under certain limits of elasticity of the material. Our approach highlights the strictly geometric concepts of the discretization process and considers the sheet formed by a flexible and inextensible material.

The interest lies in the fact that the proposed strictly geometric model of rectifying straight strips detects situations of discontinuity of the strip in anticlastic surfaces, which has not been previously foreseen. 


\section{Objectives}

Our proposal, beyond the interest of integrating a classic descriptive model into the current computational discourse, raises an important question:

Can a developable strip be obtained along a geodesic curve circumscribed to the surface to which it belongs without any kind of stretching or distortion under any circumstances?

The article tries to answer this question with numerous graphic examples. The answer (which is revealed to be negative) implies major consequences because it defines the limits beyond which it is impossible to apply the straight strip as an envelope of a freeform surface. This topic, despite being implicitly mentioned in many texts, has not been morphologically addressed in depth regarding the anticlastic surfaces. The same issue can also be posed inversely with the following question:

In what situations can a straight strip of paper circumscribed to a surface be placed without the strip encountering distortions as it is placed thereon? (Fig. 1).
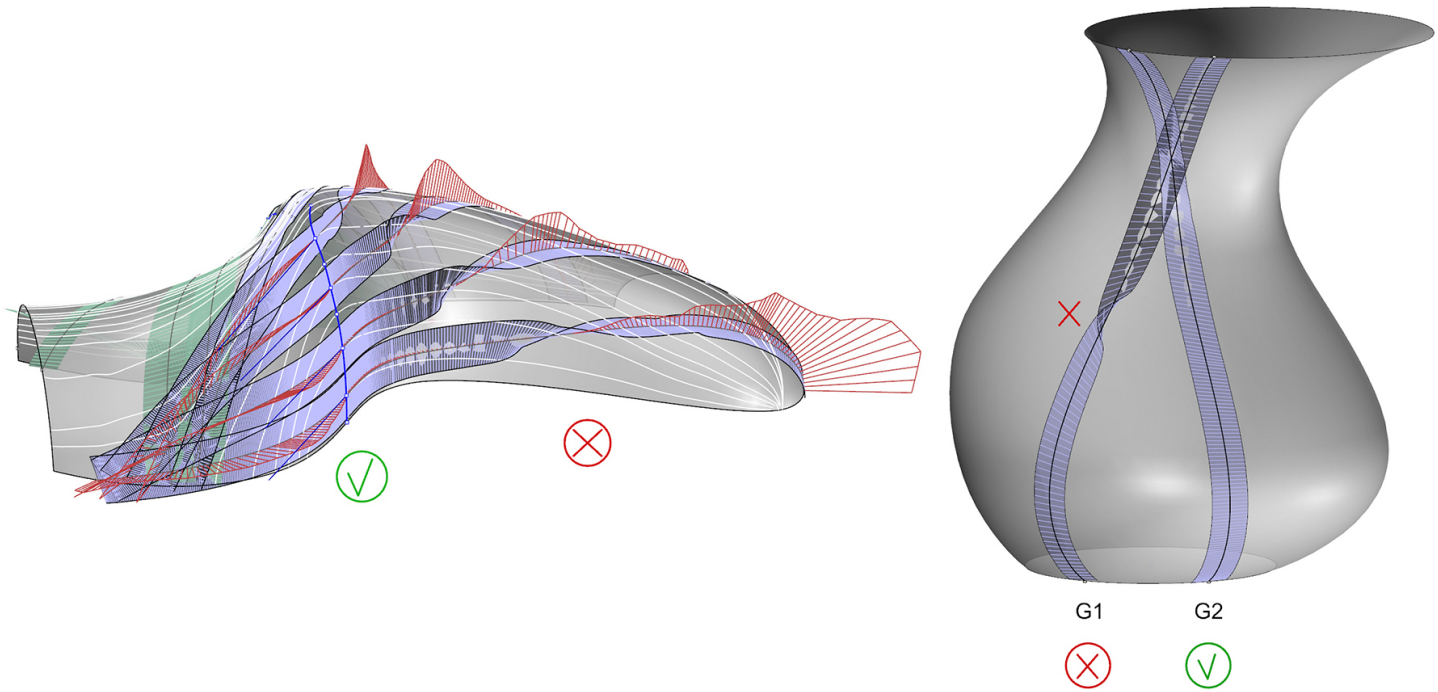

Fig. 1 Different examples of rectifying strips circumscribed to a surface

With the help of graphic tools, we reflect on the relationship between a surface, a geodesic, and its rectifying surface. Finally, we present several prototypes built in two associated laboratories, where the most practical aspect of this line of research is shown.

\section{Scope And Metodology}

We circumscribe this investigation to the use of inextensible materials, that is, to those whose fibres allow no elongation (Fig. 2). These materials are determined by a dimensional relationship where the thickness of the material is so small with respect to the other two dimensions, length and width, that it is considered negligible. The only deformation of these materials is manifested through a set of bending movements determined by a series of axes, known as the rulings of the surface, arranged continuously and infinitely close. 


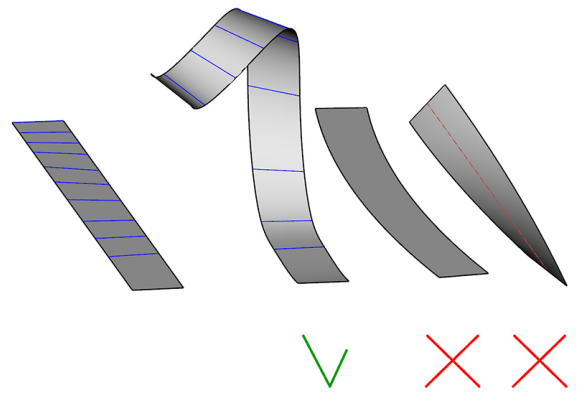

Fig. 2 Example of a strip of inextensible material. The only possible deformation of the strip is the sum of the successive flexures, which does not imply any elongation of the fibres

Digital graphic tools have allowed us to assess the various possibilities of geodesic strips and those specific situations where conflicts appear in the generation of the strip. We have worked with NURBs within the graphical environment of Rhinoceros, with the support of Grasshopper, and with AdvSurf, which is a plug-in for surface analysis (Gianpaolo 2013). We are aware that these digital tools and plugins may present certain inaccuracies in the calculation of the geodesic and the principal curvatures, which does not invalidate the result of the study, which is synthetic in nature.

\section{Process: Geometric Foundations}

Our theoretical model is based on two interrelated ideas: on the one hand, on the properties of the geodesic $\mathrm{G}$ of a surface $\mathrm{S} 1$; and on the other hand, on the concept of rectifying surface $\mathrm{R}$ of a curve $\mathrm{C}$.

According to Izquierdo-Asensi, it is known that the geodesic $\mathrm{G}$ is the "shortest path between two points A and B measured on the surface S1". On the other hand, the rectifying surface $\mathrm{R}$ of a curve $\mathrm{C}$ is "the developable surface that passes through a curve $\mathrm{C}$ which has the property of transforming said curve into a straight line $\mathrm{C}^{\prime}$ once the surface R' is deployed on a plane" (Izquierdo-Asensi 1985: 294, our trans.).

Although any curve always admits a rectifying surface, our model proposes finding the rectifying surface $\mathrm{R} 1$ from solely the geodesic curve $\mathrm{G}$ of the surface $\mathrm{S} 1$. In this way, the curve $\mathrm{G}$ is the geodesic of both the rectifying R1 and the surface S1 (Fig. 3). Thus, the geodesics provide the support of strips formed strictly of rectifying surfaces. These strips have two fundamental properties: they are straight strips when they are deployed on the plane, and they are circumscribed to the S1 surface in their spatial position.
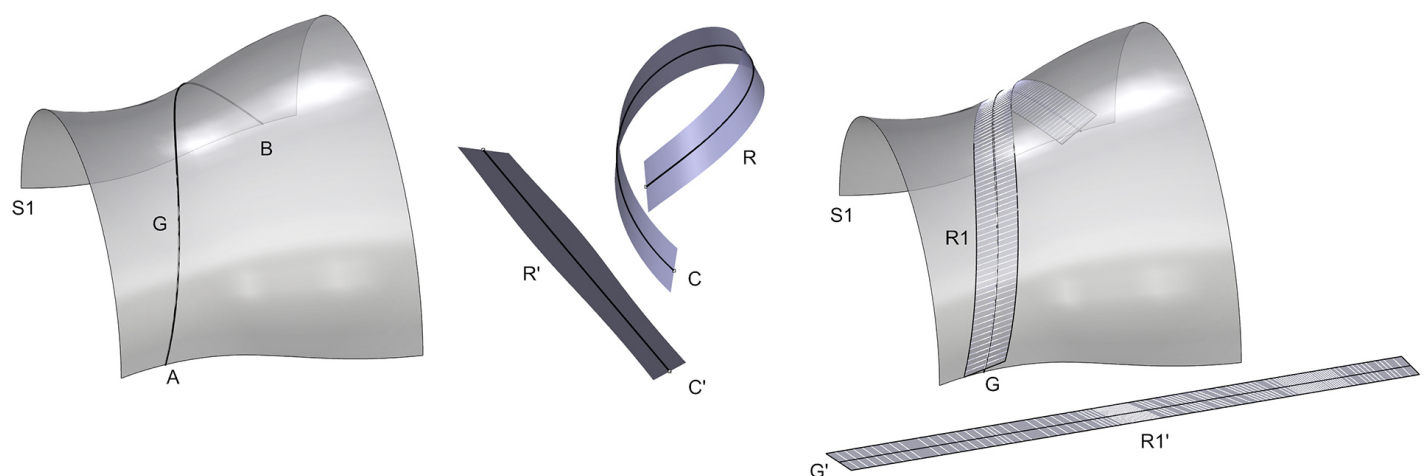

Fig. 3 Left) Geodesic G of Surface S1. Centre: Rectifying surface R of generic curve C. Right) Rectifying surface R1 of geodesic curve G 


\section{The Rectifying Surface as a Circumscribed Surface}

\section{Rectifying surface on any curve}

In Fig. 4, the three vectors that define Frenet's trihedron at each point of a curve $C$ can be observed: the tangent vector $\mathrm{T}$, the normal vector $\mathrm{N}$, and the binormal vector $\mathrm{B}$. Vectors $\mathrm{T}$ and $\mathrm{N}$ define the osculating plane, vectors $\mathrm{N}$ and $\mathrm{B}$ define the polar plane, and vectors $\mathrm{T}$ and $\mathrm{B}$ define the rectifying plane. The rectifying surface of a curve is obtained by the movement of the binormal plane, that is, by the envelope of the rectifying planes at all points of the curve (Izquierdo-Asensi 1985: 302).

The rectifying surface has the property of transforming said curve into a straight line once it is deployed on a plane. Since the rectifying surface is determined only by curve $\mathrm{C}$, and not by any associated surface, then given any curve $\mathrm{C}$, a rectifying surface can always be obtained.

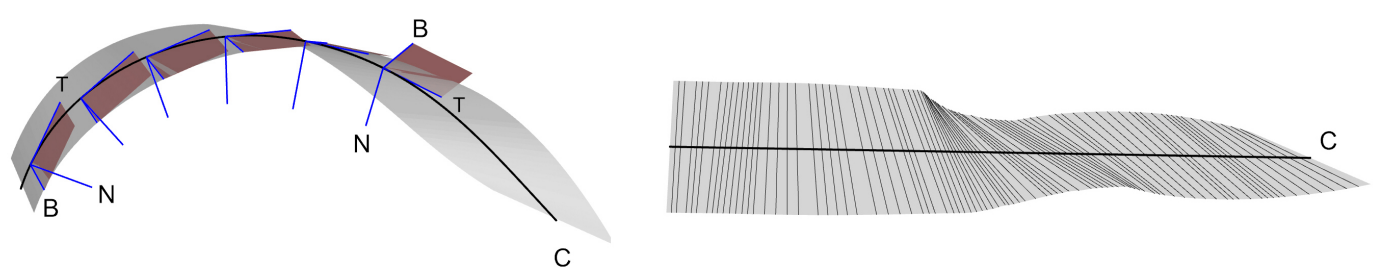

Fig. 4 Left) Frenet's Trihedron: the movement of the rectifying plane T, B, along C generates the rectifying surface. Right) Plane development of the rectifying surface where the transformed curve $\mathrm{C}$ is a straight line

\section{Rectifying surfaces understood as those circumscribed to both geodesics and surfaces}

In general, there are two canonical planes associated with a curve contained in a surface: the plane normal to the curve Nc, and the plane normal to the surface Ns. Vectors T and Nc define Frenet's trihedron, while T and Ns define Darboux's trihedron. Any curve is linked to the surface to which it belongs, which determines its geodetic curvature. Through the comparison of Frenet's and Darboux's trihedra at any point of a geodesic, we verified that the vectors $\mathrm{Nc}$ and Ns coincide, and therefore the geodesic curvature is zero (Fig. 5). Hence it is equally valid either to calculate a rectifying surface as the surface associated only with curve $G$, or to calculate it as a circumscribed surface to curve $\mathrm{G}$ and surfaces $\mathrm{S}$. 

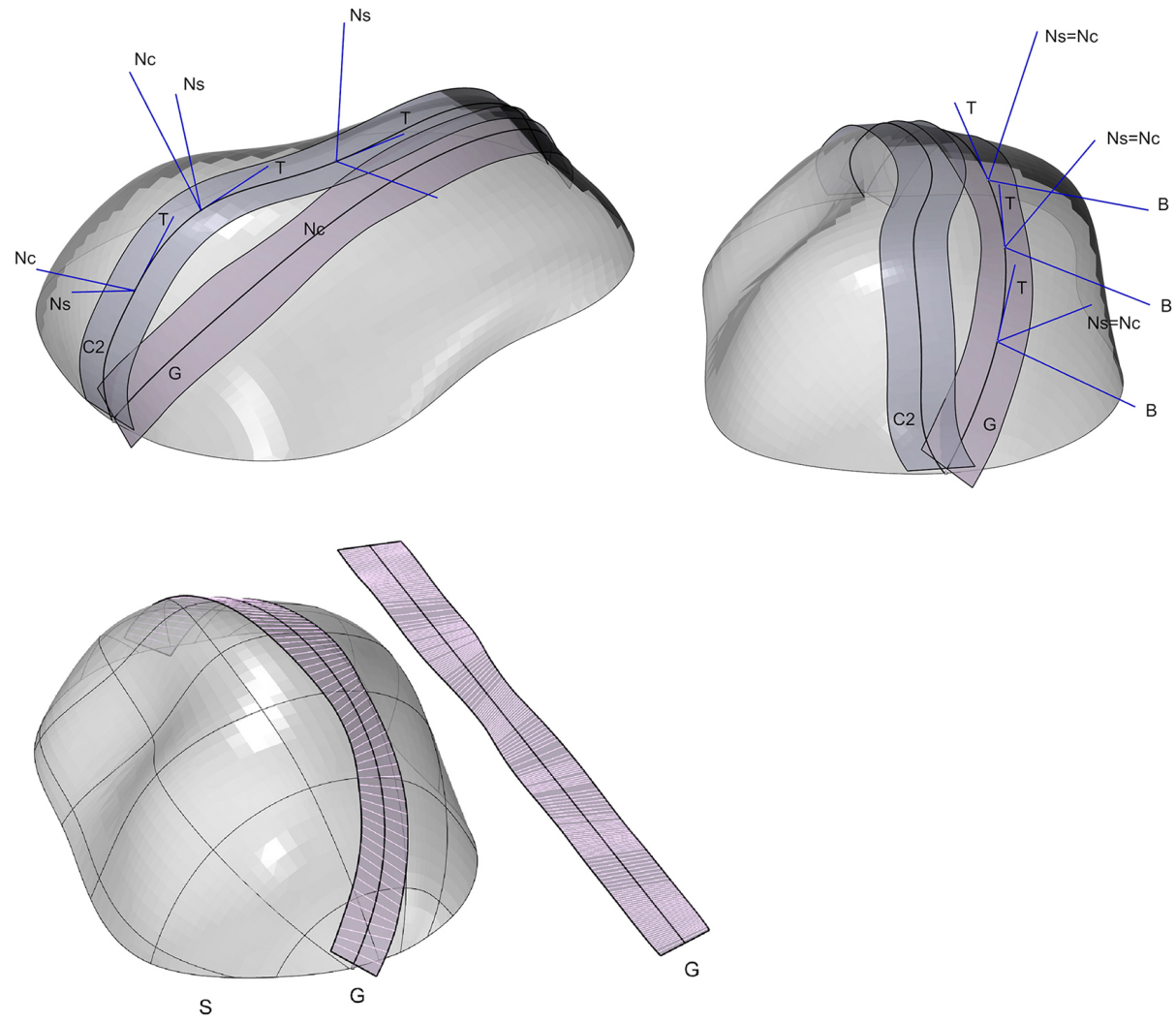

Fig. 5 Top left) Frenet's and Darboux's trihedra on any curve of a surface; Top Right) Frenet's and Darboux's trihedra for a geodesic curve; Bottom) The rectifying surface of a geodesic of $\mathrm{S}$ coincides with the surface circumscribed to $\mathrm{S}$ and its geodesic. It can be observed that the transformed curve $\mathrm{G}$ is a straight segment

\section{Results}

As a first observation of the graphic results obtained through Grasshopper algorithms, it can be stated that the rectifying strips often show discontinuities. This fact has already been highlighted in González-Quintial (2012). In this article, we delve into the reasons why these discontinuities appear in the theoretical model.

\section{First condition: Appearance of discontinuities in the strips}

In general, the model always shows discontinuity in the strip when the geodesic presents an inflection point. The discontinuity manifests itself as a twist in the rulings into a position that is impossible to achieve (Fig. 6).
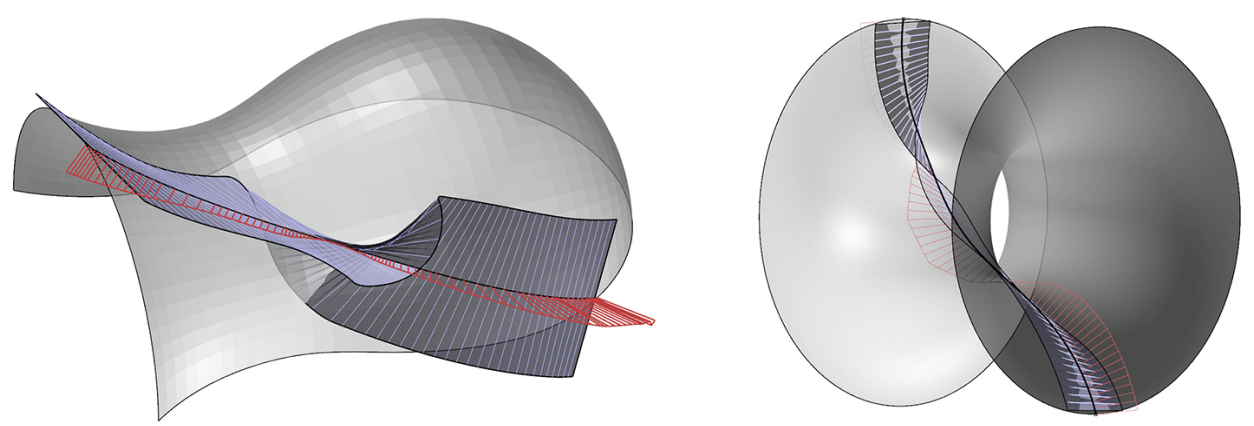

Fig. 6 Examples of discontinuities in rectifying strips 
The explanation can be found in the concept of the developable surface itself. The rectifying strip is a developed surface with two sheets, defined, like all developables, by tangential rulings on a regression line. The two tangency directions generate the two sheets of the surface. A rectifying strip affected by a regression line has one part of the strip contained in the first sheet, and the other part of the strip in the second sheet of the surface (Fig. 7). A strip without discontinuity must be contained in only one of the two sheets of the rectifying developable. This means that the regression line of the rectifying surface must not approach the curve of the strip.
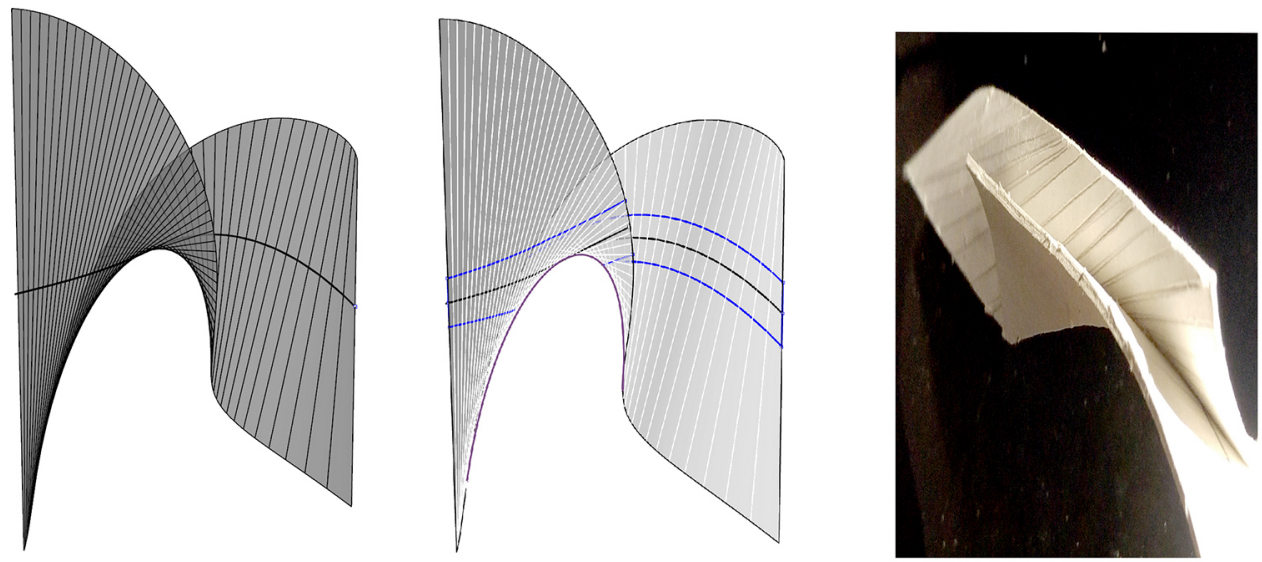

Fig. 7 Two sheets of a rectifying surface and the arrangement of the rulings

The limits that the regression line presents in the possible pure-bending movements of a strip were presented in Glaeser and Gruber (2007), who start from the rectangular flat position up to its infinite spatial positions:

When we work with torsal strips, these strips must not contain points of the edge of regression - otherwise the strip will have a self-intersection... We have written an interactive modeling computer program that allows the user to design using developable strips. It has to calculate the edge of regression and check whether the strip contains this line or not (Glaeser and Gruber 2007: 67).

From another point of view, the discontinuity presented by the inflection point has also been shown in Sprynski (2008). Other authors pay special attention to the provisions of the regulations in the two situations, which also identify such limitations (Bo and Wang 2007; Hwang and Yoon 2015).

However, our approach to the problem is not focused on studying the possible purebending movements of an existing strip, but on choosing which geodesic curves on a surface can support straight strips that are free of discontinuities.

Wallner et al. (2010) addresses the discretization of surfaces using straight or "almost straight" strips with two methods: The first, known as "developed tangent", uses strips tangentially circumscribed to the surface along geodesic curves. This first method starts from the same geometric premises as those shown in this article. However, the method is developed with the notion of conjugate tangents, a completely different procedure from that of rectifying surfaces. It is not strange that, as occurs with the appearance of discontinuities in our method, the "tangent developable" procedure conflicts with 
certain geodesic points: "Unfortunately this [tangent developable method] does not work in practice. One reason is that the rulings of the tangent developables may behave in weird ways" (Wallner et al. 2010: 80).

In a search for a satisfactory solution to this problem, Wallner et al. propose a second method of strip generation based on the binormal vectors of the geodesic points. The binormal method consists of drawing a set of binormal vectors to the geodesic curve that will be the rulings of the strips. In this new strip there is no conflict in the twist of the rulings in the vicinity of the inflection point. In short, a new surface that is different from the rectifying surface will be created (Fig. 8).

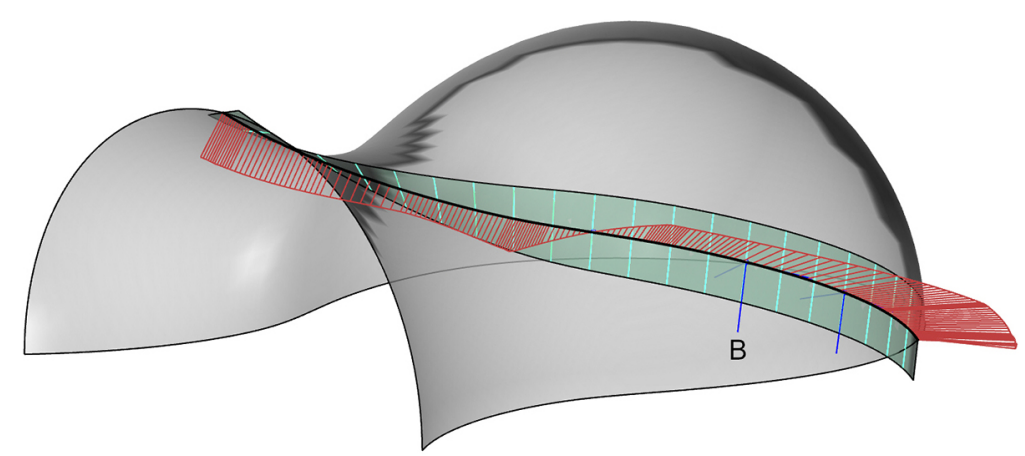

Fig. 8 The Binormal Method

The strips obtained are not developable surfaces but rather warped ruled surfaces. In order to reach the final position from an initial flat position, it is necessary to assume a certain capacity of elongation in the fibres: the isometry between the initial and final strip disappears when considering a certain elasticity of the material. As the authors themselves argue: "From a mathematical viewpoint, it [the "binormal" method] is a simple and obvious way of defining panel surfaces, but it is unclear that this surface should be the shape of a panel after it has been forced to follow a geodesic on the surface" (Wallner et al. 2010: 83).

The problem of the validity of the binormal model with other assumptions, which involves the twist capacity of the strip in its length/width relationship, is openly manifested when the strip becomes wider. If architectural envelopes with very wide strips are to be generated, then this method, which is useful in gridshells, is not operational. The theoretical model of pure bending, or of non-stretchable fibre sheet materials, becomes more appropriate, despite the thorny question of strip discontinuities that it entails: an issue we strive to elucidate later in the paper.

\section{Second condition: Appearance of very oblique rulings on the strips}

As a second observation of the graphic results, oblique rulings appear on the strips when the geodesic tends to be a straight line or when it has zones of almost zero curvature (Fig. 9, left).

On ruled surfaces, it can be observed that when the geodesic approaches the direction of the rulings, then their inclinations become more oblique. In the limit situation, where strip rulings and surface rulings coincide, the width of the strip tends towards zero 
(except in special cases). Geodesics on warped ruled surfaces offer the general case in which to observe this characteristic. The other situation of oblique rulings in the strip is manifested when the geodesic, even presenting a single curvature, has a zone where it tends towards zero.
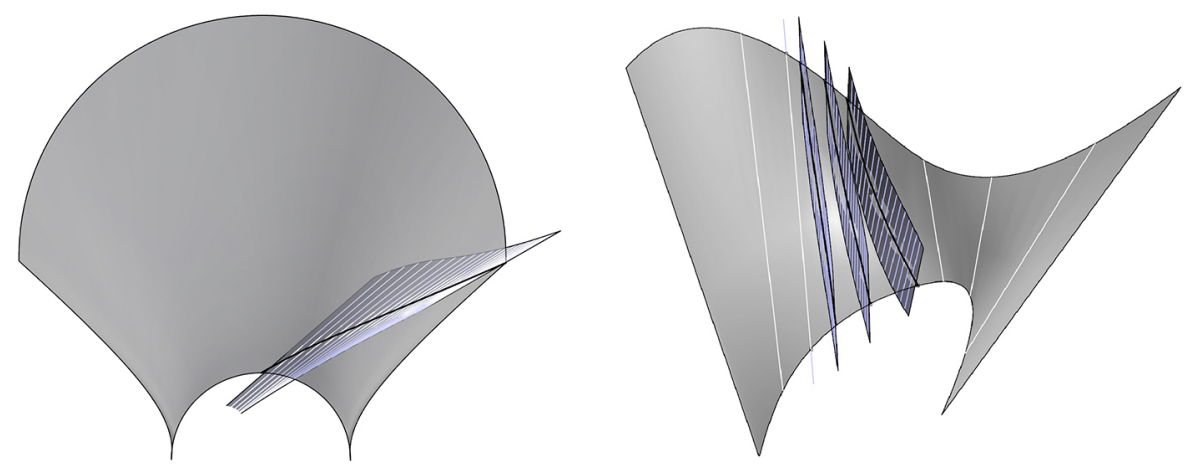

Fig. 9 Examples of the appearance of very oblique rulings on the strips

The width of the strip is considerably reduced due to the inclinations of the rulings of the strip. The cause is the presence of the regression line, but unlike the previous case, now the regression line does not cut the axis of the strip. It maintains a certain proximity and always remains on one side of the axis. The rulings do not fully rotate; they only rotate to the axis direction, thereby causing the strip to narrow. Beyond a certain angle of twist, it can be observed that is no longer possible to widen the strip (Fig. 10).
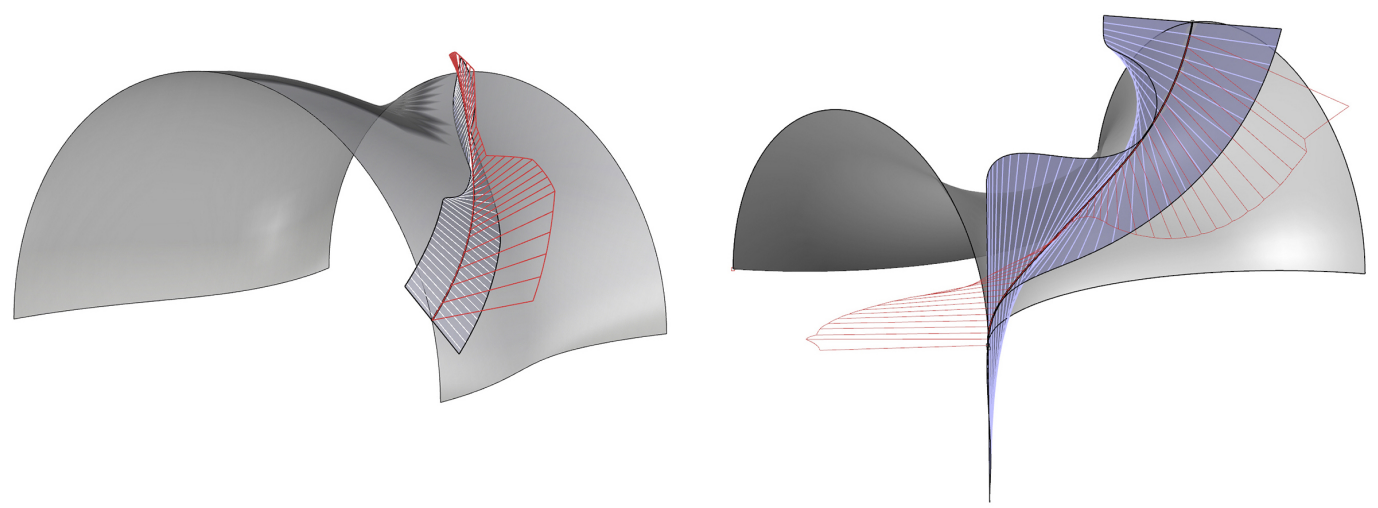

Fig. 10 Examples of narrowness in the rectifying strip due to the inclinations of the rulings

\section{Discussion}

\section{Strips with a controlled inflection point}

By refocusing the problem for the assumption of inextensible materials, we have shown how the inflection point in the geodesic produces discontinuity in the strip. However, there are exceptional situations where this does not occur, which we have called the "controlled inflection point". What condition determines that some geodesics show discontinuity in the strip and others do not? 
Among all the geodesics with an inflection point, those that do not offer discontinuity in their strip are those that, at their inflection point, have the tangent vector oriented with the main direction of zero curvature of the surface (Fig. 11).
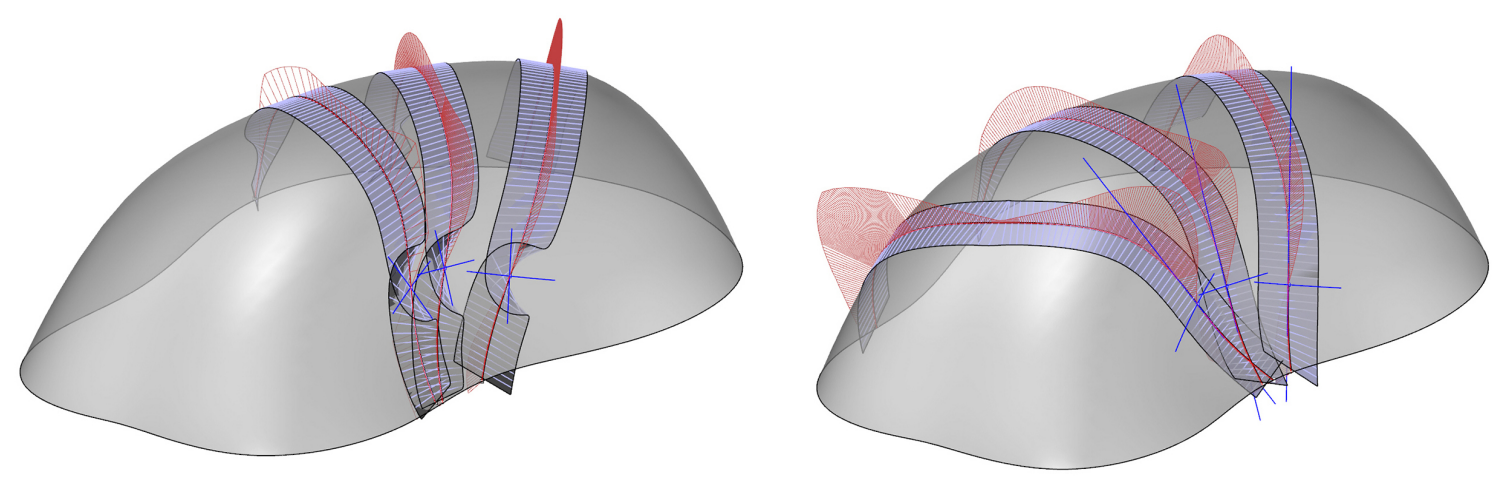

Fig. 11 Left) Rectifying strips that show discontinuity on geodesics with inflection points; Right) Rectifying strips that do not show discontinuity on geodesics with inflection points

We justify this property with the concept of curvature lines of a surface. The work with developable strips on principal curvature lines has been studied in Yang et al. (2006) and Pottmann et al. (2008), and this question has been sufficiently displayed: Any strip circumscribed to two curvature lines $D$ of the surface Swill always be developable. If the separation distance between these two curvature lines tends towards zero, then the surface of the strip will rest, at the limit, on a single curvature line. We can trace a surface $\mathrm{T}$ circumscribed to the surface $\mathrm{S}$ by said curvature line. A strip contained in $\mathrm{T}$ that is resting on the curvature line does not show discontinuity at any point, including the conflicting inflection points that now coincide with the parabolic points on the surface.

Therefore, if a geodesic $\mathrm{G}$ of the surface $\mathrm{S}$ passes through a parabolic point $\mathrm{P}$ of the surface and its tangent at that point is oriented in the direction of the main curvature of the surface, then both curves, geodesic and main curvature, tend to be coincident near point $\mathrm{P}$. If the strip supported on the main curvature line shows no discontinuity, then neither does the strip that supports the geodesic (Fig. 12). In this way, the geodesics, the curvature lines and the parabolic points on the surface can be graphically related.

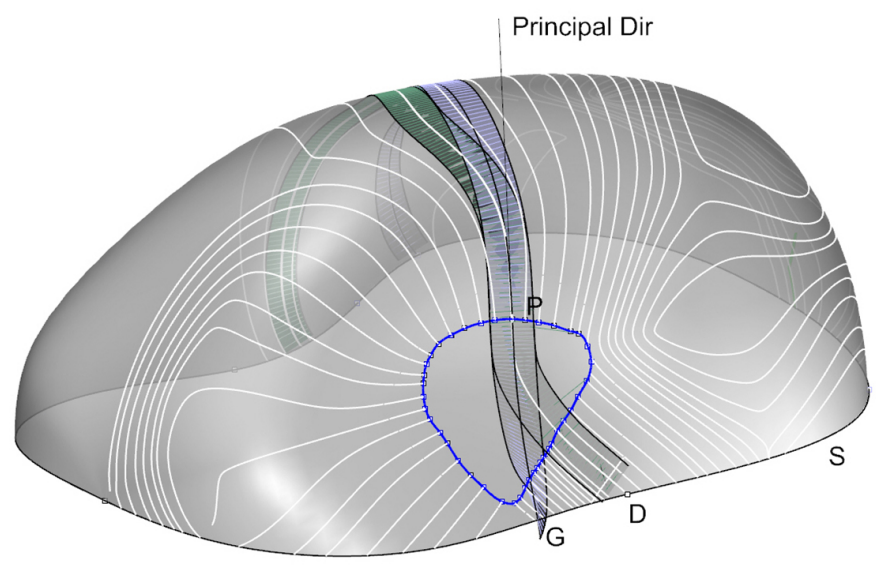

Fig. 12 The curvature lines of surface $\mathrm{S}$, where the parabolic points of the surface and the main direction of zero curvature at point $\mathrm{P}$ are indicated in blue 


\section{The problem of choosing geodesics}

Based on the examples presented, the first criterion for choosing optimal geodesics must be to find geodesics that have a single curvature and are not close to zero at any of their points. However, the shape of the geodesic is highly conditioned by the nature of the surface, whether it be synclastic, anticlastic, or mixed.

Any geodesic traced onto a synclastic surface is always free of inflection points. Therefore, synclastic surfaces can be discretized by rectifying straight strips. The problem of discontinuity of the strip appears in anticlastic surfaces: those whose two curvatures have an opposite sign. In these surfaces, the geodesic can present inflection points and the strips can show greater narrowing problems.

A freeform surface is generally a mixed surface made up of synclastic and anticlastic surfaces. The parabolic points, those of zero Gaussian curvature, determine the transition between these surfaces. All dented surfaces feature synclastic and anticlastic surface areas, separated by a line of parabolic points. The inflection points of the geodesic can appear precisely at the parabolic points of the surface or in the anticlastic zone.

The area of zero Gaussian curvature of the mixed surface can be crossed by geodesics without inflection points and by geodesics with inflection points. This fact is of great interest since geodesics with a controlled inflection point can be chosen, thereby producing a deterministic discretization model.

\section{Discretization of freeform surfaces: Geometric restrictions}

Two strategies are employed here to address the discretization of any freeform surface: either the choice of a range of conflict-free geodesics, or the strict use of geodesics with controlled inflection point.

1. Discretization by means of conflict-free rectifying strips.

Geodesics that have a single curvature that is not close to zero at any of its points will accept the rectifying strip without problems of discontinuity or narrowness. This enhances a wide variety of strip solutions. In Fig. 13, the optimal range of geodesics to cross the zero Gaussian curvature zone of the surface can be observed.

- Discretization using rectifying strips with a controlled inflection point. This is a deterministic method, where all strips have controlled inflection points. The design process can be parameterized in Grasshopper, in the following steps (Fig. 14).

- Determine the main curvature lines of the surface.

- Determine the inflection points of the main curvature lines. These will be parabolic points of the freeform surface.

- Plot two geodesic curves (in both directions, left and right) from the inflection point of the curvature line of the surface in the main direction of curvature. 
- As a result, two geodesics are attained that follow opposite paths from a parabolic point on the surface. These two geodesics can be understood as a single curve since they are joined tangentially at the starting point.

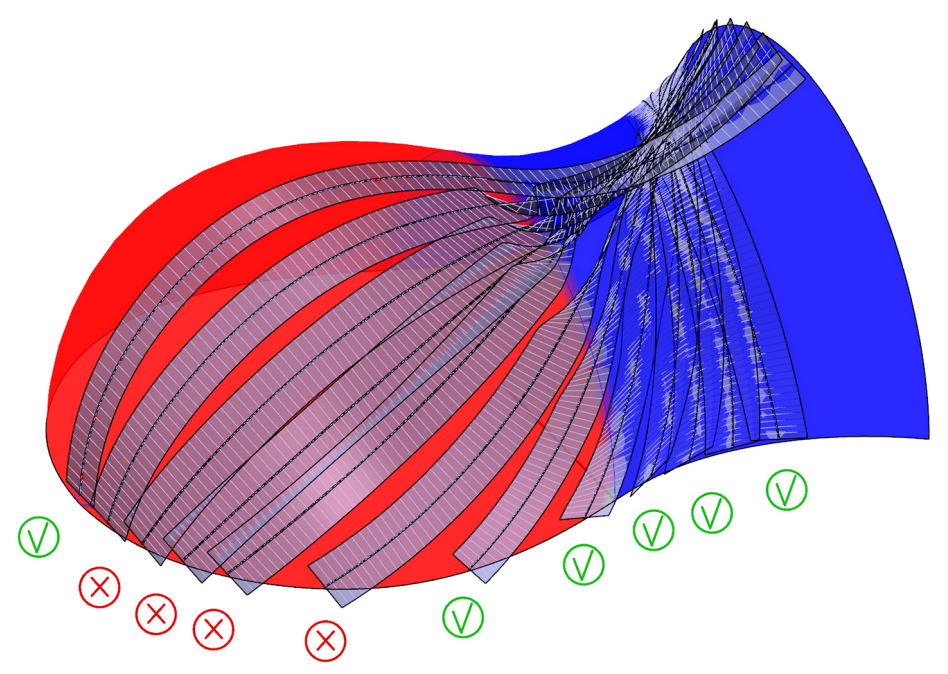

Fig.13 Valuation of appropriate strips to discretize a revolution surface

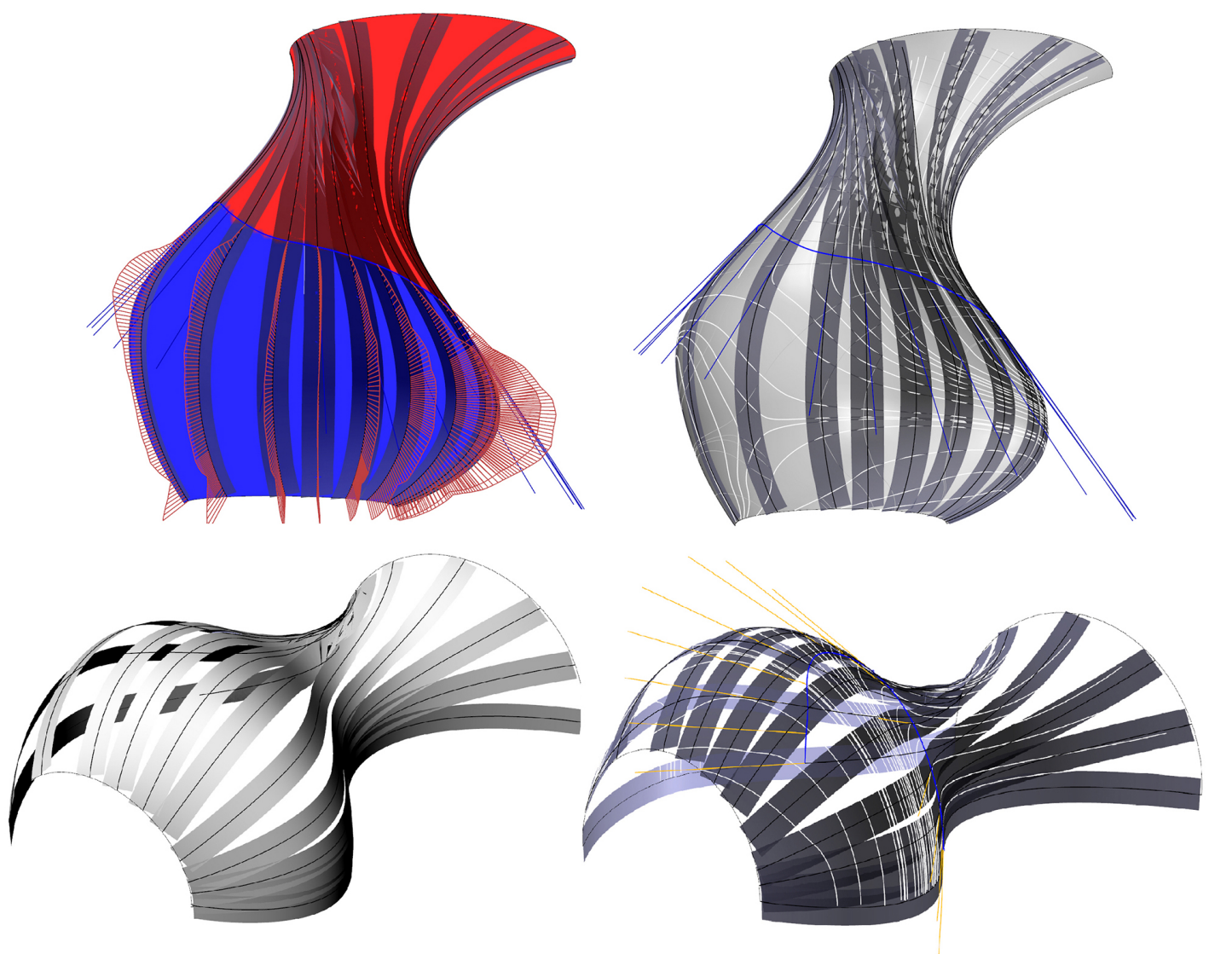

Fig. 14 Example of discretization using rectifying strips on geodesics with a controlled inflection point on a freeform surface 
There are limitations in the controlled inflection point method. It is not possible to adjust the rectifying straight strip if the geodesic has more than one inflection point. Adjusting the orientation of the geodesic to the main direction at the first point, the strip is misaligned if a second inflection point appears (Fig. 15).
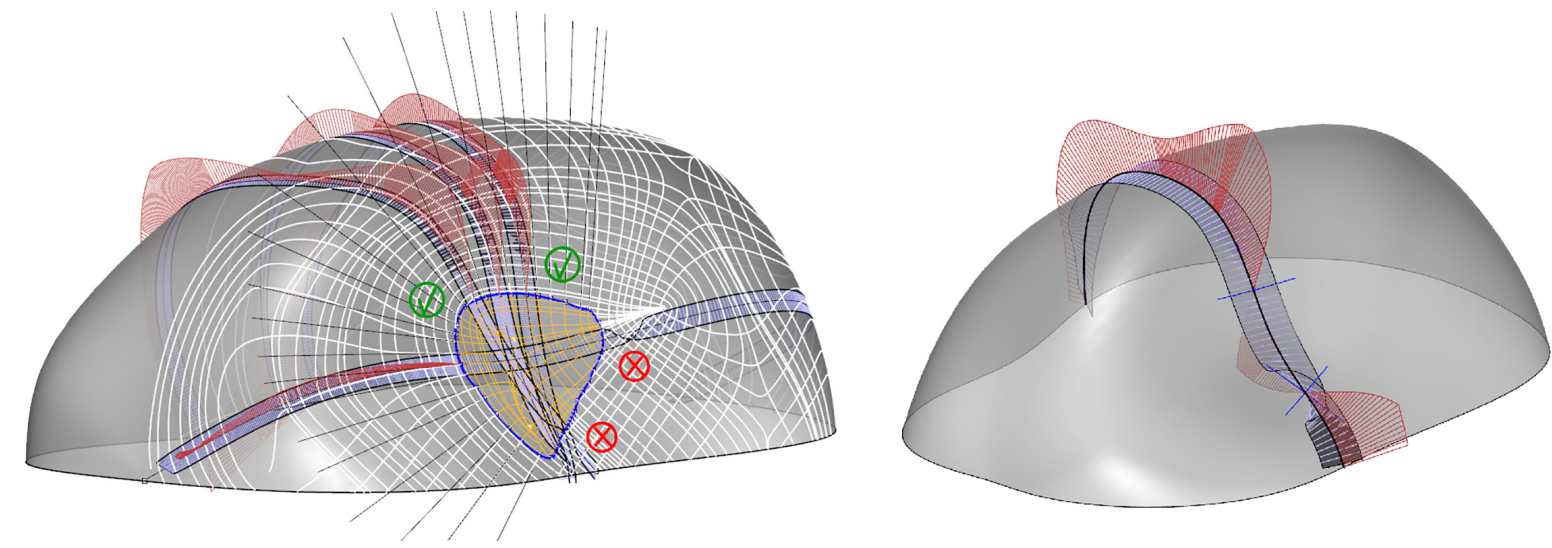

Fig. 15 When the geodesic presents two inflection points it is impossible to adjust the rectifying strip in the second

\section{Application: Approach to the discretization of double curvature surfaces using rectifying developable strips}

This research has been made possible through the collaboration of two laboratories: Fablab Donostia ETSA-AGET UPV-EHU and Geometry Digital Lab-ETSIE-US. Over recent years, we have developed several prototypes of discretized surfaces using straight strips on geodesics. The research originated from a known structural model, used in gridshells, which involved weaving a surface using narrow strips following geodesic paths on surfaces. This initial model evolved towards wider strips with the desire to study the possibilities that geodesics offer in the panelling of architectural surfaces. The successes and failures, in terms of the lack of correspondence between the theoretical models and the behaviour of the material, have been decisive for the development of this article and the rectifying strip theoretical model.

Regarding the possible uses and application of these rectifying straight strips, these can be used not only as the geometric basis for the design and construction of purely cladding elements, but also as structural elements arranged as gridshells. The outcomes from this research are focused on self-supporting structural models that form continuous shells. Specifically, in the case of structural prototypes some FEM analyses have been carried out where the timber elastic behaviour has been taken into account. The structural behaviour is mainly based on the form of the surface working as a shell. From a geometric point of view, the structural behaviour of the shell is conditioned by the disposition of the holes over the surface, and above all by the amount of stiffness induced by the connections at the cross points between the strips, and by how the strip confers shear resistance to the shell. These models show, in the same way as do prototypes, that the free border of the shell should be strengthened in order to increase the stiffness of the shell along these free edges. 
In the first studies, the Thorus I/Thorus II projects use narrow strips on toric surfaces where there are clearly differentiated positive and negative curvature zones, and special attention is paid to the anticlastic zone of the surface (Fig. 16, left and centre).

The prototype (Fig. 16, right) was discretized by means of two families of geodesics upon which narrow interwoven strips were supported. It showed problems in the vicinity of the inflection point and could therefore not be completed.
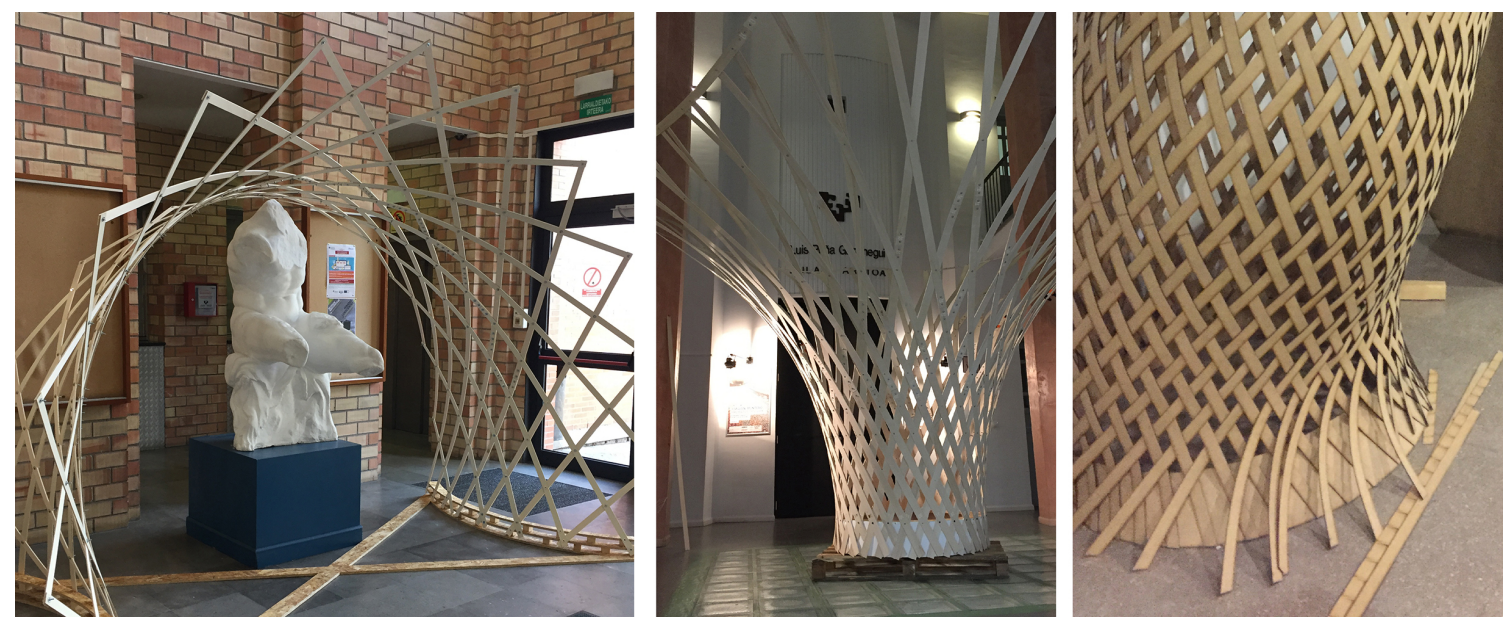

Fig. 16 Left) Thorus I; Centre) Thorus II; Right) Prototype, failure in inflection area

Other work addressed the discretization of minimal surfaces: in those a main curvature of zero means that the two main curvatures are equal and opposite in sign at each point on the surface. These surfaces are anticlastic and very efficient from the structural point of view. A number of these projects addressed the construction of trilobed and tetralobed surfaces and employed geodesics with rectifying strips drawn thereon (Fig. 17 , left and centre).

The scHerk pavilion aimed to scale up the tests carried out on the minimal surface models discretized with rectifying surfaces on geodesics. In this prototype, we proposed a double structural layer based in this property: a second developable surface can be generated from the initial one, by offsetting it along the normal vectors, which maintains the isometry between the two surfaces (Fig. 17, right).
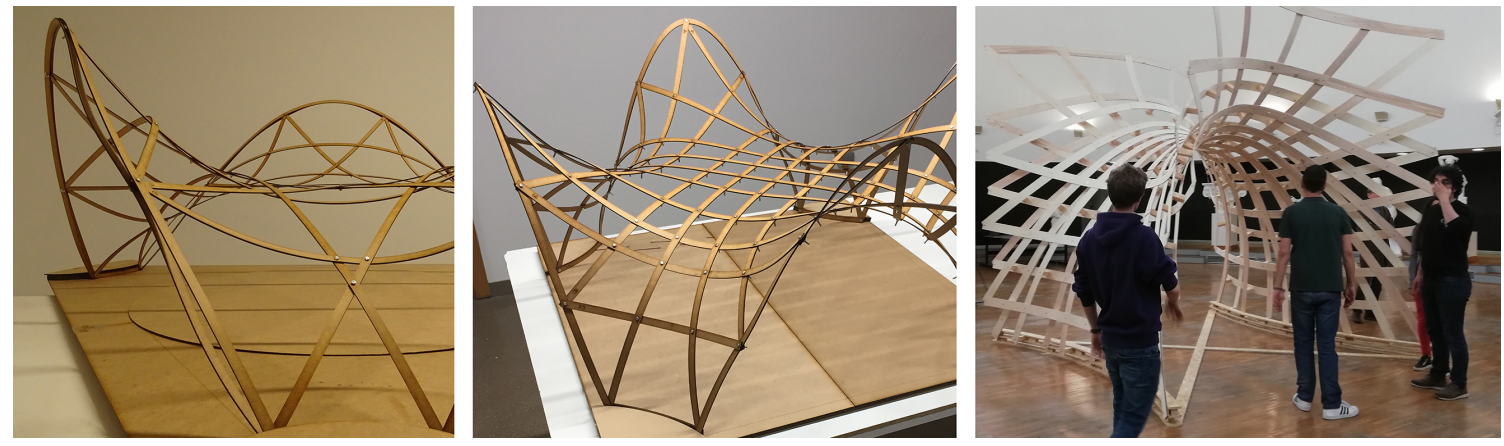

Fig. 17 Left and centre) Prototypes; Right) scHerk pavilion 
In the Jacaranda project (named in honour of Felix Candela's project for Club Jacaranda), wider strips were introduced. The strips showed bending problems at the inflection points of the geodesics. An attempt was made to overcome this situation by using a very flexible sheet material. The search for the origin of such discontinuities has led us to the geometric rectifying strip model presented in this article (Fig. 18, left).

In the Geodesic-Catenoid project, we address the discretization of the catenoid with wide strips free of discontinuities. The catenoid is a minimal surface generated by the revolution of a catenary around a coplanar axis. We designed an algorithm to control the inflection points in the geodesic tracing and the rulings in the strips. Finally, the surface was discretized by geodesics without a inflections point, thus avoiding continuity problems in the strips (Fig. 18, centre and right).
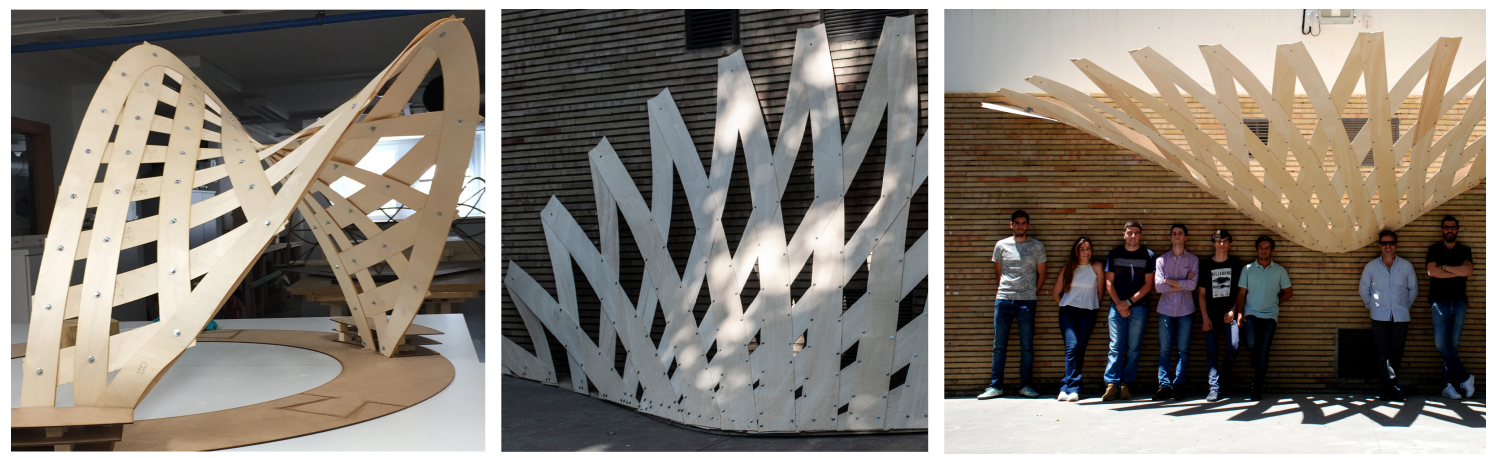

Fig. 18 Left) Jacaranda Project; Centre and Right) Geodesic-Catenoid Project

In certain cases, minimal surfaces have been used as the base geometry of the shells thanks to their appropriate structural behaviour. They have also been used as a way of exploring their geometric properties, as the derivatives of their asymptotic curves or of the optimal relationship that exists between the dimension of the surface and the volume it encloses. All these questions deserve further study and provide valid lines for future research.

\section{Conclusions}

We present a graphic model, complementary to others, to discretize surfaces using strips, which has a wide application in architecture. The model presents the possibilities of covering a surface tangentially by means of developable straight strips, formed by a flexible and inextensible material.

Synclastic surfaces always accept straight developable strips in any of their geodesics, but anticlastic and mixed surfaces depend on the inflection points of the chosen geodesic.

Inextensible strips do not present inconsistencies in the following cases:

1. Along a geodesic that presents a single curvature that is not close to zero at any of its points;

2. Along a geodesic that presents a controlled inflection point;

3. Along a geodesic that coincides, at all its points, with a main curvature line. 
The theoretical model proposed is based on the "sheet of paper concept". This means that only the pure bending of the strip is allowed, and not the lengthening of the fibres. Despite the use of plywood in the construction of the prototypes following the approximation to these models, the fibres of the plywood allow a certain degree of this type of lengthening, and therefore a certain discrepancy arises between the theoretical and physical models. The tensional requirements to which the strips are submitted, apart from that of their own weight, are those from the active bending action introduced by the bending process. Currently, an extension of this research is being carried out for the general characterisation of an elastic model based on real deformations measured by using photogrammetric data gathering in order to more accurately measure the differences between the theoretical and virtual model and the physical model.

\section{Acknowledgements}

This research has been partially funded by internationalization grants to the Instituto Universitario de Arquitectura y Ciencias de la Construcción (IUACC) of the VI Own Research and Transfer Plan of the University of Seville, the Department of Architecture of the University of the Basque Country and the Housing Department of the Basque Government. All images are by the authors.

\section{References}

Bo, Pengbo and Wenping Wang. 2007. Geodesic-controlled developable surfaces for modeling paper bending. Computer Graphics Forum 26(3): 365-374. https://doi.org/10.1111/j.1467-8659.2007.01059.x

Chen, Sheng Gwo. 2010. Geodesic-like curves on parametric surfaces. Computer Aided Geometric Design 27(1): 106-117. http://dx.doi.org/10.1016/j.cagd.2009.10.001

Deng, B., H. Pottmann and J. Wallner. 2011. Functional webs for freeform architecture. Eurographics Symposium on Geometry Processing 30(5): 1369-1378. https://doi.org/10.1111/j.1467-8659.2011.02011.x

Deregibus, Carlo and Mario Sassone. 2008. Mathematical and structural properties of geodesic curves: An application on a free form gridshell. Journal of the International Association for Shell and Spatial Structures 49(3): 159-166.

Do Carmo, M. 1976. Differential Geometry of Curves and Surfaces. Prentice-Hall.

Gianpaolo, Savio. 2013. AdvSurf Version 0.1 (C) Rhino Open Projects - June 2013.

Glaeser, Georg and Franz Gruber. 2007. Developable surfaces in contemporary architecture. Journal of Mathematics and the Arts 1(1): 59-71. https://doi.org/10.1080/17513470701230004

González-Quintial Francisco, Javier Barrallo and Ana Artiz-Elkarte. 2015. Freeform surfaces adaptation using developable strips and planar quadrilateral facets. Journal of Facade Design and Engineering 3: 57-90. https://doi.org/10.3233/FDE-150033

González-Quintial, F. 2012. Método de adaptación de formas de doble curvatura mediante superficies desarrollables, Ph.D. Thesis, EHU-UPV.

Harding, John, Will Pearson, Harry Lewis and Stephen Melville. 2014. The Ongreening Pavilion. Advances in Architectural Geometry 2014, eds. Philippe Block, Jan Knippers, Niloy J. Mitra, Wenping Wang, 295-308. Springer. https://doi.org/10.1007/978-3-319-11418-7 
Hwang, Hae Do and Seung Hyun Yoon. 2015. Constructing developable surfaces by wrapping cones and cylinders. CAD Computer-Aided Design 58: 230-235. http://dx.doi.org/10.1016/j.cad.2014.08.025

Izquierdo-Asensi, Fernando. 1985. Geometría Descriptiva Superior y Aplicada. Madrid: Dossat.

Jiang, Caigui, Klara Mundilova, Florian Rist, Johannes Wallner and Helmut Pottmann. 2019. Curve-pleated Structures. ACM Transactions on Graphics 38(6). https://doi.org/10.1145/3355089.3356540

Jung, Amaury, Stefanie Hahmann, Damien Rohmer, Antoine Begault, Laurence Boissieux and Marie-Paule Cani. 2015. Sketching Folds: Developable Surfaces from Non-Planar Silhouettes. ACM Transactions on Graphics 34 (5): 155. https://doi.org/10.1145/2749458

Kahlert, Joe, Matt Olson and Hao Zhang. 2011. Width-bounded geodesic strips for surface tiling. Visual Computer 27(1): 45-56.

Kilian, Martin, Simon Floery, Zhonggui Chen, Niloy J. Mitra, Alla Sheffer and Helmut Pottmann. 2008. Curved Folding. ACM Transactions on Graphics 27(3). https://doi.org/10.1145/1360612.1360674

Kilian, Martin, Aron Monszpart and Niloy J. Mitra. 2017. String actuated curved folded surfaces. ACM Transactions on Graphics 36(3). https://doi.org/10.1145/3015460

Lai, Shuhua and Fuhua Frank Cheng. 2011. Approximate geodesics on smooth surfaces of arbitrary topology. Computer-Aided Design and Applications 8(4): 499-506. https://doi.org/10.3722/cadaps.2011.499-506

Lawrence, Snežana. 2010. Developable Surfaces: Their History and Application. Nexus Network Journal 13(3): 701-714. https://doi.org/10.1007/s00004-011-0087-z

Pirazzi, Claudio and Yves Weinand. 2006. Geodesic lines on free-form surfaces Optimized grids for timber rib shells. 9th World Conference on Timber Engineering 2006, WCTE 2006, Vol. 1: 72-78.

Pottmann H., A. Asperl, M. Hofer and A. Kilian. 2007. Architectural Geometry. Bentley Institute Press.

Pottmann, Helmut, Alexander Schiftner, Pengbo Bo, Heinz Schmiedhofer, Wenping Wang, Niccolo Baldassini, and Johannes Wallner. 2008. Freeform surfaces from single curved panels. ACM Transactions on Graphics 27(3). https://doi.org/10.1145/1360612.1360675

Pottmann, Helmut, Qixing Huang, Bailin Deng, Alexander Schiftner, Martin Kilian, Leonidas Guibas and Johannes Wallner. 2010. Geodesic patterns. $A C M$ SIGGRAPH 2010, SIGGRAPH 2010. https://doi.org/10.1145/1778765.1778780

Ravi Kumar, G.V.V., Prabha Srinivasan, Holla V. Devaraja, K. G. Shastry and B. G. Prakash. 2003. Geodesic curve computations on surfaces. Computer-Aided Geometric Design 20(2): 119-133. https://doi.org/10.1016/S0167-8396(03)00023$\underline{2}$

Rose, Kenneth, Alla Sheffer, Jamie Wither, Marie-Paule Cani and Boris Thibert. 2007. Developable Surfaces from Arbitrary Sketched Boundaries. SGP '07 - 5th Eurographics Symposium on Geometry Processing, July 2007, Barcelona, Spain, 163-172.

Soriano, Enrique. 2017. Low-tech geodesic gridshell: Almond Pavilion. ArchiDOCT, 4(2): 29-40. 
Sprynski, N., N. Szafran, B. Lacolle and L. Biard. 2008. Surface reconstruction via geodesic interpolation. CAD Computer-Aided Design 40(4): 480-492. https://doi.org/10.1016/j.cad.2008.01.005

Surazhsky, Vitaly, Tatiana Surazhsky, Danil Kirsanov, Steven J. Gortler and Hugues Hoppe. 2005. Fast exact and approximate geodesics on meses. ACM Transactions on Graphics 24(3): 553-560.

Taibo-Fernández, Ángel. 1983. Geometría Descriptiva y sus aplicaciones, Tomo II. Madrid: Tébar Flores.

Tang, Chengcheng, Pengbo Bo, Johannes Wallner and Helmut Pottmann. 2016. Interactive design of developable surfaces. ACM Transactions on Graphics 35(2): 12. https://doi.org/10.1145/2832906

Wallner, Johannes, Alexander Schiftner, Martin Kilian, Simon Flöry, Mathias Höbinger, Bailin Deng, Qixing Huang and Helmut Pottmann. 2010. Tiling freeform shapes with straight panels: algorithmic methods. Advances in Architectural Geometry 2010. https://doi.org/10.1007/978-3-7091-0309-8_5

Yang, Liu, Helmut Pottmann, Johannes Wallner, Yong-Liang Yang and Wenping Wang. 2006. Geometric Modeling with Conical Meshes and Developable Surfaces. ACM Transactions on Graphics 25(3): 681-689. https://doi.org/10.1145/1141911.1141941 